\title{
Beitrag zur Kenntnis des Sklerödems Erwachsener.
}

\author{
Von \\ Oberstabsarzt z. D. Dr. Roscher (Berlin).
}

Ein Fall von Sklerödem, den ich kürzlich einige Zeit beobachten konnte, verdient bei der Seltenheit der Erkrankung kurze Beschreibung.

Der 22 $\frac{1}{2}$ Jahre alte Mann, von Beruf Maurer, bemerkte als Soldat plötzlich eine Behinderung bei Kopf- und Armbewegungen und fühlte, da $B$ die Haut sich spannte und straff und derb war. Am 3. November 1910, etwa 3 Tage nachdem ihm dieser Zustand zuerst aufgefallen war, bekam ich den Kranken zu Gesicht. Er gab an, sein Vater leide seit dem Feldzug 1870/71 an Rheumatismus in beiden Beinen, seine Mutter und 5 Geschwister seien am Leben und gesund, in der Verwandtschaft seien irgendwelche bemerkenswerten Krankheiten nicht vorgekommen. Er selbst habe im Anschluß an Diphtherie im Alter von 17 Jahren eine Lähmung beider Beine gehabt, deren Heilung eine $3 / 4$ jährige Behandlung erforderte, im übrigen sei er stets gesund gewesen, mit Ausnahme einer 3 Monate zurückliegenden Erkrankung an weichem Schanker, welcher bei Lazarettbehandlung schnell geheilt war. Vor dem Eintritt des jetzigen Leidens sei er, wie er mit aller Bestimmtheit angibt, völlig wohl gewesen; dagegen habe er mehrere Tage, nachdem er die Steifigkeit bemerkt hatte, etwas Kopfschmerzen und erhöhte Temperatur (bis $37^{\circ}$ ) gehabt und auck einen Bläschenausschlag an der Nase bekommen; er habe dagegen Aspirin genommen und sei in zwei Tagen wieder vollkommen wohl gewesen.

Bei der Untersuchang fand sich der folgende Befund: Mittelgrober, kräftig gebauter, gut genährter, muskulöser Mann. Die Haut des Oberkörpers ist glatt, etwas matt und wachsfarben. Bei genauerem Zusehen fällt eine eigenartige Spannung der Hautoberflüche auf, die Felderung 
tritt sehr deutlich hervor. Die Follikel sind gut erbalten, die Haare und Nägel in jeder Beziehung ohne Besonderbeiten.

Das Gesicht ist etwas maskenartig starr, das Minenspiel minimal. Am linken Nasenflügel befindet sich ein anscheinend von einem Herpes herrührender Schorf.

Im übrigen sieht die Haut unverändert aus, insbesondere bestehen weder Gefäßerweiterungen noch Pigmentierungen.

Bei der Betastung zeigt die befallene Haut eine diffuse derbe Infiltration und zwar symmetrisch an Kopf, Hals, Brust, Rücken und Oberarmen, weniger stark ausgesprochen am Bauch und den oberen $2 / 3$ der Unterarme. Die Haut füblt sich derb und prall an, läßt sich nur an den Unterarmen, an der Innenseite der Oberarme sowie am Bauch und zwar nur in dicken Falten abheben; an Gesicht, Hals, Brust, Rücken und der AuBenseite der Oberarme ist sie nur etwas und über den Jochbogen überhaupt nicht verschieblich. Stirnrunzeln ist nicht möglich, der Mund kann nur wenig geöffnet werden, die Nasolabialfalten treten beim Zähnezeigen kaum hervor. Die Augenlider sind ziemlich starr, die Lidspalten schmal. Die Konturen der Gelenke und der normalen Gruben z. B. über den Schlüsselbeinen sind verwaschen, der Hals ist plump und walzenförmig.

Die Bewegungen des Kopfes sind nach allen Richtungen stark behindert und zwar die Nickbewegung stärker als die Drehung, welche noch bis zu einem Winkel von etwa $40^{\circ}$ möglich ist.

Die Ausdehnungsfähigkeit des Brustkorbes bei der Atmung ist sichtlich vermindert, die Differenz zwischen gröBter Ein- und Ausatmung beträgt $6 \mathrm{~cm}$; der ganze Brustkorb fühlt sich prall, wie gepanzert an.

Die Arme können ziemlich ausgiebig bewegt, die Hände ohne große Mühe in den Nacken geschlagen werden. Die Grenzen der Hautinfiltration sind äußerlich nicht sichtbar, aber durch Palpation als ziemlich scharf festzustellen. In der Grenzzone fühlt man besonders deutlich, daß die Infiltration in den tieferen Schichten der Haut gelegen ist. Die unter der Haut gelegenen Organe wie Knochen, Drüsen usw. sind nicht durchzufühlen.

Die derbe Haut läßt sich ein wenig eindrücken, ohne daß aber eine deutliche Delle zurückbleibt. Der Druck ist nirgends schmerzhaft, wie überbaupt alle subjektiven Empfindungen und ¡irgendwelche Entzündungserscheinungen vollkommen fehlen.

Die Venen treten an den Unterarmen auch beim Erheben wie gestaut hervor, während sie an den Oberarmen, obwohl deutlich sichtbar, nicht das Niveau überragen und in abtastbaren Furchen verlaufen.

An den Händen, den unteren Extremitäten von den Glutäen abwärts und den Genitalien ist die Haut vollkommen normal; sie ist lebhaft marmoriert, am ganzen Körper zeigt sich auf Streichen ein starkes und langdauerndes Nachröten, keine Urticaria factitia. 
Die Lidbindehäute und sichtbaren Schleimhänte sind frei, auch das Schlucken ist unbehindert. Die Lymphdrüsen sind, soweit tastbar, nicht vergrößert.

An den sich gut ausdehnenden Lungen, an Leber und Milz läßt sich krankhaftes nicht nachweisen, der Urin ist frei von Eiweiß, Zucker und geformten Elementen. Die Pupillen- und Sehnenreflexe sind normal auszulösen. Die Sensibilität ist für alle Qualitäten am ganzen Körper gleichmäßig erhalten.

Leichte Veränderungen zeigten sich nur am Herzen:

Der Spitzenstob und die Herzgrenze reicht links bis zur Brust. warzenlinie. Der 1. Ton an der Spitze ist akzentuiert und klappend, der 2. Pulmonalton ist verstärkt. Orthodiagraphisch stellen sich die Herzgrenzen wie folgt:

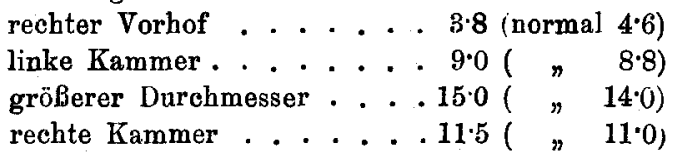

Der Blutdruck beträgt, an beiden Oberarmen gemessen, $125 \mathrm{~mm}$ Quecksilber nach Riva-Rocci, ist also nicht wesentlich erhöht, besonders wenn man in Betracht zieht, daß die Kompression der derben Haut mehr Druck erfordert als unter normalen Verbältnissen.

Ein Unterschied in der Schweiß - und Talgdrüsensekretion an den kranken und gesunden Stellen besteht nicht. Die v. Wassermannsche Blutprobe ist negativ.

Die Temperatur hat $37 \cdot 2$ nie überschritten.

Auffallend war die dauernd fast maximale Erweiterung der Pupillen; es interessierte daher zu untersuchen, ob dies auf eine Reizung der vom Sympathikus innervierten Dilatatoren zurückzuführen, der Mann also zu der Gruppe der Sympathikotoniker zu zählen war. Für diese Annahme ließ sich ein Beweis nicht erbringen, denn es trat auf Traubenzuckerdarreichung and Injektion von $1 \mathrm{mg}$ Suprarenin Zuckerausscheidung nicht ein und es wurde auf 1 cg Pilokarpin reichliche Schweiß- und Speichelabsonderung erzielt.

Zur histologischen Untersuchung wurde ein keilförmiges Stück aus der linken Schulterhaut heraus geschnitter. Der Schnitt klaffte weit, ließ sich kaum durch die Naht, welche infolge der Spannung bald durchschnitt, vereinen und heilte durch Granulation recht allmählich zu.

Die Untersuchung, welche Herr Privatdozent Dr. A r n d t so freundlich war zu kontrollieren, ergab völlig normale Beschaffenheit der Epidermis und der obersten Kutisschichten; in den mittleren und tiefen Teilen der Kutis waren die Bindegewebsbündel durch weite Spalträume auseinander- 
gedrängt. Diese Lücken zeigen sich größtenteils leer, an einzelnen Stellen aber mit einer kaum gefärbten durchsichtigen Masse gefüllt. Im übrigen bestanden durchaus normale Verhältnisse, an Pigment und elastischen Fasern keine Veränderungen, keine Entzündungserscheinungen, nur um die Gefäße herum eine geringe Ansammlung kleiner mononukleärer Zellen, die sich aber in so geringen Grenzen hält, daß von einer Entzündung nicht gesprochen werden kann.

Die Behandlung bestand in heißen, bzw. Dampfbädern und nachfolgender Massage mit 1\% iger Thiosinaminsalbe, später in Fibrolysininjektionen.

Der Erfolg der Behandlung war bis Ende Dezember 1911 ein geringer und auch als sich Anfang Februar 1912 der Mann mir wieder vorstellte, konnte ich eine wesentliche Veränderung nicht feststellen. Immerhin ist die Haut etwas geschmeidiger und verschieblicher gèworden und kann über den Unterkieferästen, den Schultern und den Seitenteilen der Brust in dicken Falten erhoben werden; auch beim Runzeln der Stirn zeigen sich seichte Querfurchen und die Nasolabialfalten treten beim Zähnezeigen etwas dentlicher hervor; aber die Beweglichkeit des Kopfes hat sich nur wenig gebessert, die der Arme eher etwas vermindert. Das Erheben der Arme gelingt nur bis zu einem Winkel von etwa $140^{\circ}$ und das Zurückschlagen der Hände in den Nacken erfolgt nur mit Mühe.

Subjektiv bestand dauernd völliges Wohlbefinden bei gut erhaltenem Kräftezustand; außer über Beschränkung der Bewegungen und Hautspannung wurden Klagen nicht geäußert, auch bei körperlichen Anstrengungen bestand Atemnot nicht, nur das Gefühl, als ob die Kleidung zu eng wäre.

Das Krankheitsbild und der Krankheitsverlauf entsprechen durchaus dem Sklerödem Erwachsener, wie es zuerst von Buschke, dann von Rissom und Nobl beschrieben und auch von Blaschko und Bettmann beobachtet worden ist. ${ }^{1}$ ) Es handelt sich auch hier um ein anscheinend akut entstandenes und rom Kopf ausgegangenes, diffus und streng symmetrisch den Oberkörper ergreifendes, tiefes pralles Ödem der Haut mit langdauerndem Verlauf,

1) Die beiden von Pinkus veröffentlichten Fälle weichen nach Beginn und Verlauf von den übrigen ab und stellen jedenfalls keine reinen Fälle von Sklerōdem dar. 
ohne Beteiligung der Epidermis und oberflächlichen Kutisschichten und ohne Neigung zu sekundären Veränderungen. Allerdings ist in meinem Falle das letztere noch nicht mit Sicherheit auszuschließen, ebenso wie die Rückbildung zur Norm noch abgewartet werden muß, ehe die Diagnose vollkommen erhärtet ist.

Über die Ätiologie der Krankheit ergibt der vorliegende Fall keine Aufklärung. Während nach den bisherigen Berichten das Leiden sich im AnschluB an Infektionskrankheiten (Influenza, epidemische Parotitis, Scharlach) entwickelte, muß es im Gegensatz hierzu bei unserem Kranken als ausgeschlossen gelten, daß eine Infektionskrankheit unmittelbar vorausgegangen ist. Das anfänglich bestehende, mit leichtem Fieber verbundene Unwohlsein, dessen Überstehen auch noch nachträglich durch den Herpes erwiesen war, spricht vielleicht für einen fieberhaften Beginn der Erkrankung, wenn es mit dieser überhaupt in Zusammenhang steht, kann aber bei der Geringfügigkeit und dem schnellen Schwinden der Beschwerden als Ausdruck einer Infektion wohl ohne weiteres nicht angesprochen werden. Als Ursache im Sinne der Auffassung eines infektiōsen Ursprungs bliebe nur die allerdings 5 Jahre zurückliegende, aber mit schweren Lähmungen verbundene Diphtherie und es sei daher der Möglichkeit Ausdruck gegeben, daß vielleicht eine diphtherische Schädigung der die Hautgefäße innervierenden Nerven für die Entstehung des Leidens in Betracht kommt, ähnlich wie $R$ issom in seinem Fall eine Lymphstockung infolge krankhafter Störung der Innervation der Hautgefäße angenommen hat. Eine Erkrankung der Lymphgefäße entsprechend der Theorie Buschke-Senator konnte histologisch nicht erwiesen werden. 


\section{Literatur.}

Buschke. Über Sklerödem. Berliner klin. Wochenschr. 1902.

Pinkus. 2 Fälle von Sklerödem. Dermatolog. Zeitschrift. 1907.

Rissom. Beitrag zur Kenntnis des Sklerödercs des Erwachsenen. Archiv für Dermatologie und Syphilis. 1909. Bd. XCIV.

Nobl. UÜber das benigne Sklerödem Erwachsener. Wiener mediz. Wochenschrift. 1909.

Bettmann, Blaschko. Verhandlungen des X. DermatologenKongresses. 